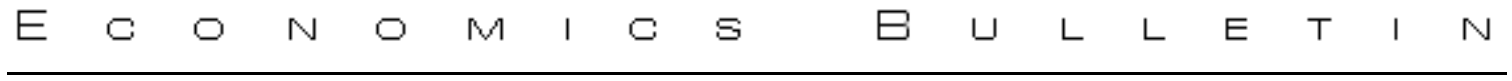

\title{
Preemption, Start-Up Decisions and the Firms' Capital Structure
}

\author{
Michele Moretto \\ University of Padova and FEEM
}

\author{
Paolo M. Panteghini \\ University of Brescia and CESifo
}

\begin{abstract}
In this article, we analyse the interactions between financial and start-up decisions in an oligopolistic framework, where firms compete to enter a new market. We show that preemption can substantially reduce the negative effects of credit rationing on start-up investment decisions.
\end{abstract}

\footnotetext{
The authors would like to thank Gianni Amisano, Giacinta Cestone, Domenico Cuoco, Paolo Fulghieri, Søren Bo Nielsen, and an anonymous referee for helpful comments and suggestions. They are responsible for any remaining errors.

Citation: Moretto, Michele and Paolo M. Panteghini, (2007) "Preemption, Start-Up Decisions and the Firms' Capital Structure." Economics Bulletin, Vol. 4, No. 39 pp. 1-14

Submitted: May 14, 2007. Accepted: October 17, 2007.

URL: http://economicsbulletin.vanderbilt.edu/2007/volume4/EB-07D90003A.pdf
} 


\section{Introduction}

It is a well-known fact that start-up firms usually fear that a rival may seize their business advantage by moving first. This entails the possibility that the firm's project value is sharply reduced by potential competitors. Another important feature of start-ups is the limited access to external sources. ${ }^{1}$ As far as we know, however, only a few papers have studied the interaction between entry decisions and capital structure.

In this paper we therefore look at the effects of limited access to external finance on start-up investments. Using an oligopolistic framework with preemption and incomplete information, we will then compare an all-equityfinancing setup with a benchmark optimal leverage one. The former entails a 100\% credit constraint, whereas the latter implies full financial flexibility.

This paper is linked to two streams of literature. The first concerns real options and market structure. As start-ups can be optimally timed, option pricing techniques are a useful tool to analyse these decisions. ${ }^{2}$ Furthermore, since the second half of the 90 s there has been a growing interest in integrating strategic considerations with option pricing techniques. ${ }^{3}$ Most of the published articles, however, assume complete information: this is clearly unsatisfactory in the case of start-ups, where information on the characteristics and behaviour of competitors is scarce. A notable exception is Lambrecht and Perraudin (2003) (hereafter LP), who studied the interactions between entry decisions and preemption in a duopoly model with incomplete information. ${ }^{4}$ However, they assumed that firms are all equity-financed. ${ }^{5}$

The second line of research is the one on optimal capital structure. As

\footnotetext{
${ }^{1}$ One relevant exception were internet stocks whose hypervaluation led to a relatively easy access to external finance (see e.g. Ottoo, 2001).

${ }^{2}$ This is one of the main contributions of the real-option theory, which basically says that "when the future is highly uncertain, it pays to have a broad range of options open" (Coy, 1999). Graham and Harvey (2001) show that about 25\% of the companies surveyed always or almost always incorporate real options when evaluating a project. Furthermore, McDonald (2000) and Jagannathan and Meter (2002) argue that even when firms use standard techniques, they may apply ad hoc rules of thumb which proxy for optimal timing behaviour.

${ }^{3}$ See e.g. the books by Huisman (2001) and Smit and Trigeorgis (2004).

${ }^{4}$ Other exceptions are Grenadier (2002) for the Cournot-oligopolist case, and Moretto (2000), Dosi and Moretto (2007) for the second-mover advantage.

${ }^{5}$ Lambrecht (2001) analyses the impact of debt financing in a real option leader-follower model. However he assumes full information.
} 
we know, debt financing entails both costs and benefits. On the one hand, debt may lead to bankruptcy and agency conflicts between shareholders and debtholders. On the other hand, debt not only ensures relevant tax benefits (Leland, 1994) but can also reduce agency conflicts between managers and shareholders (Jensen, 1986). Under such a model, therefore, firms choose their capital structure optimally, by weighing the costs and benefits of debt financing. It is worth noting however that most existing papers do not consider how strategic behaviour may affect a firm's capital structure choice. ${ }^{6}$

In this article, we aim to provide a natural extension of these studies, by analysing the interactions between financial and start-up decisions in an oligopolistic framework with preemption, where firms compete to enter a new market. We will prove that preemption makes all-equity-financed investment strategies closer to those undertaken under optimal leverage. This means that preemption can substantially reduce the discouraging effects of credit rationing on start-up investment decisions.

The article is structured as follows. Section 2 introduces the model. Section 3 studies the interactions between financial and real choices. Section 4 concludes.

\section{The model}

Let us focus on an oligopolistic market where $N+1(N>0)$ firms compete under the fear of preemption. To exploit new market opportunities, each firm must pay a sunk cost $I_{n}, n \in N+1$. Risk is fully diversifiable and the risk-free interest rate $r$ is given. By assumption, if one of the firms invests, the others lose any opportunity to invest. ${ }^{7}$ Once investment is undertaken, the project cannot be abandoned, although the firm's operation can be temporarily and costlessly suspended when its payoff falls to zero (see Dixit and Pindyck, 1994).

The firms' payoffs $\Pi_{t}$ evolve according to the following geometric Brownian motion

$$
d \Pi_{t}=(r-\delta) \Pi_{t} d t+\sigma \Pi_{t} d z_{t}, \quad \text { with } \Pi_{0}=\Pi,
$$

\footnotetext{
${ }^{6}$ Exceptions are Mauer and Ott (2000) and Miao (2005). However, they do not model the threat of preemption.

${ }^{7}$ In this article we assume that no incumbent exists. However, the quality of results would not change if we assumed a leader-follower setup. For details on this point, see Moretto (2000).
} 
where $\delta \geq 0$ is the net "dividend" yield (see e.g. McDonald and Siegel, 1985), $\sigma$ is the instantaneous standard deviation and $d z_{t}$ is the increment of a standard Wiener process.

Following LP we assume that $I_{n}$ is the firm $n$ 's private information. At each instant, firm $n$ observes the realisation of the state variable $\Pi_{t}$, and, according to its private information on $I_{n}$ and its conjectures on the rivals' strategies, it decides whether to invest or not. ${ }^{8}$ This entails an optimal stopping time decision where firm $n$ invests if its payoff $\Pi_{t}$ reaches a trigger value $\bar{\Pi}_{n}$, above which investment is profitable. Since $\bar{\Pi}_{n}$ depends on $I_{n}$, it is private information as well. ${ }^{9}$ To determine a firm's investment strategies, we also assume that $\bar{\Pi}_{n}$ is drawn from the conditional distribution function $F_{n}\left(\bar{\Pi}_{n} ; \Pi\right)$, with a continuous differentiable density $f_{n}\left(\bar{\Pi}_{n} ; \Pi\right)$ on the support $\left[\bar{\Pi}^{l}, \bar{\Pi}^{u}\right) .{ }^{10}$ With no loss of generality, we also assume $F_{n}=F, \forall n \in N+1$.

As time passes, $\Pi_{t}$ is expected to rise. If, therefore, competitors have not yet invested, each firm learns that its rivals' trigger points lie in a smaller higher interval. A sufficient statistic that captures this information is given by $U_{t}=\sup _{0 \leq s<t}\left(\Pi_{s}\right)$ which denotes the maximum level of payoff up to time $t$ without one of the firms having invested. This means that, at any instant $t \geq 0$, each firm updates its conditional distribution of the rivals' trigger values according to Bayes' rule

$$
F_{t}\left(\bar{\Pi} ; U_{t}\right)=\frac{F(\bar{\Pi} ; \Pi)-F\left(U_{t} ; \Pi\right)}{1-F\left(U_{t} ; \Pi\right)} \quad \text { where } U_{0}=\Pi \text {, }
$$

which is strictly increasing in the $\left[U_{t}, \bar{\Pi}^{u}\right.$ ) interval. Given (2), a firm learns by observing its rivals' behaviour. This leads to a Bayesian Nash equilibrium, consisting of $N+1$ linked "stopping time problems", where each firm solves its own investing problem, conditional on rivals' actions. If, therefore, no competitor has yet invested, the game goes on; otherwise, it ends up. ${ }^{11}$

\footnotetext{
${ }^{8}$ Notice that the quality of results does not change if we assume incomplete information on firm-specific profitability.

${ }^{9}$ As shown by LP, asymmetric information on costs results in the optimal trigger value $\bar{\Pi}_{n}$ being, under the same regularity condition, a unique continuous increasing mapping function of $I_{n}$, i.e., $\bar{\Pi}_{n}=\bar{\Pi}\left(I_{n}\right)$ with $\frac{\partial \bar{\Pi}_{n}\left(I_{n}\right)}{\partial I_{n}}>0$.

${ }^{10}$ We assume that $\bar{\Pi}_{n}$ does not have any mass at $\Pi$, but it does have positive density there. Therefore, the set $\left\{\bar{\Pi}_{n} \mid f_{n}\left(\bar{\Pi}_{n} ; \Pi\right)>0\right\} \subseteq\left[\bar{\Pi}^{l}, \bar{\Pi}^{u}\right)$ has a density function with positive support for $\bar{\Pi}^{l} \leq \Pi$ and $\bar{\Pi}^{u} \leq \infty$. For further details see LP.

${ }^{11}$ With no loss of generality, we assume that if all firms invest simultaneously, each firm has a probability $1 /(N+1)$ of winning the race.
} 
Let us finally focus on the capital structure. In order to measure the effects of debt constraint, we will compare the all-equity-financing case, where firms cannot borrow, with a trade-off case where leverage is optimally chosen. For simplicity, we assume no debt renegotiation.

Following Leland (1994), we define $C$ as the coupon paid to debtholders and assume that the benefit from debt financing is proportional to the coupon paid, i.e. $\varepsilon C$ with $\varepsilon>0$. Moreover, we assume that default takes place when $\Pi_{t}$ drops to $C .^{12}$ In this case, equityholders are expropriated by lenders. Such an expropriation causes a sunk cost $v C$ with $v>0{ }^{13}$

\section{The firms' decisions}

If real and financial decisions are made simultaneously, the firm's objective function is equal to the maximum between zero and its option to invest. The firm $n$ 's problem at time $t \geq 0$ is thus one of choosing the optimal entry trigger $\bar{\Pi}_{n}$ and the coupon $C$, namely,

$$
O_{n}\left(\Pi_{t}, U_{t} ; \bar{\Pi}_{n}, C\right)=\max _{\bar{\Pi}_{n}>0, C>0}\left\{0, V_{n}\left(\Pi_{t} ; \bar{\Pi}_{n}, C\right) \operatorname{Pr}\left(T_{n}<T_{N-n} \mid U_{t}\right)\right\}, \quad \forall n
$$

where $V_{n}\left(\Pi_{t} ; \bar{\Pi}_{n}, C\right)$ is the option to invest for a given trigger $\bar{\Pi}_{n}$. When firms can borrow, $V_{n}\left(\Pi_{t} ; \bar{\Pi}_{n}, C\right)$ is equal to the sum of the equity value, called $E_{n}\left(\Pi_{t}, C\right)$, and the value of debt, i.e., $D_{n}\left(\Pi_{t}, C\right)$.

The term $\operatorname{Pr}\left(T_{n}<T_{N-n} \mid U_{t}\right)$ measures the probability that firm $n$ moves first. Instant $T_{n}=\inf \left(t>0 \mid \Pi_{t}=\bar{\Pi}_{n}\right)$ is the expected stopping time at which firm $n$ finds it optimal to invest and $T_{N-n}=\inf (t>0 \mid$ $\left.\Pi_{t}=\min _{\forall i \neq n}\left(\bar{\Pi}_{i}\right), i \in N+1\right)$ is the minimum investment timing of its $N$ competitors. Assuming that each firm's investment trigger is independently distributed, and applying rule (2), we obtain $\operatorname{Pr}\left(T_{n}<T_{N-n} \mid U_{t}\right)=$ $\left[1-F\left(\bar{\Pi}_{n} ; \Pi_{t}\right) / 1-F\left(U_{t} ; \Pi_{t}\right)\right]^{N}$. Given these assumptions we can show that:

\footnotetext{
${ }^{12}$ This results in debt being protected (see Leland, 1994). However, the quality of results does not change if we assume that debt is unprotected, i.e., that equityholders can choose when to default.

${ }^{13}$ This cost may be justified by the fact that not only default procedures are per se costly and time-consuming but also by the fact that the law may make it difficult for lenders to repossess collateral (see e.g. Panteghini, 2007a).
} 
Proposition 1 The firm n's optimal trigger point under debt-financing is:

$$
\bar{\Pi}_{n}^{D}=\frac{G\left(\bar{\Pi}_{n}^{D} ; N\right)}{1+m} \delta I_{n} \quad \forall n
$$

where $G\left(\bar{\Pi}_{n}^{D} ; N\right) \equiv \frac{\beta_{1}+h\left(\bar{\Pi}_{n}^{D}, N\right)}{\beta_{1}-1+h\left(\bar{\Pi}_{n}^{D}, N\right)}, m \equiv \frac{\beta_{2}}{\beta_{2}-1} \delta \frac{\varepsilon}{r}\left(\frac{1}{1-\beta_{2}} \frac{\varepsilon}{\varepsilon+r \nu}\right)^{-1 / \beta_{2}}>0$ and $h\left(\bar{\Pi}_{n}^{D} ; N\right) \equiv \frac{N \bar{\Pi}_{n}^{D} f\left(\bar{\Pi}_{n}^{D} ; \Pi\right)}{1-F\left(\bar{\Pi}_{n}^{D} ; \Pi\right)}$ is the hazard rate, with $\beta_{1}>1$ and $\beta_{2}<0$. The optimal ratio between $C$ and $\bar{\Pi}_{n}^{D}$ is given by:

$$
\frac{C}{\bar{\Pi}_{n}^{D}}=\left(\frac{1}{1-\beta_{2}} \cdot \frac{\varepsilon}{\varepsilon+r \nu}\right)^{-\frac{1}{\beta_{2}}} .
$$

Proof. See Appendix A.

Proposition 1 shows the joint effect of preemption and leverage on a firm's strategies, and thus it is an extension of previous work in two respects. On the one hand, the introduction of debt-financing allows us to generalise LP's findings. On the other hand, the introduction of preemption also improves Mello and Parsons (1992) and Gamba et al. (2005). In particular, (4) shows that the hazard rate affects the option multiple $G\left(\bar{\Pi}_{n}^{D} ; N\right)$ : it is easy to show that the higher the hazard rate, the smaller the multiple $G\left(\bar{\Pi}_{n}^{D} ; N\right) .{ }^{14}$

The effect of leverage on investment decisions is measured by the term $\frac{1}{1+m}<1$. It is clear that $\frac{\partial m}{\partial \varepsilon}>0$ and $\frac{\partial m}{\partial \nu}<0$. This means that the greater the benefit of borrowing $(\varepsilon),{ }^{15}$ the lower the firm's trigger point, i.e., the greater the firm's propensity to invest at any given time. The reverse is true when the cost of default is accounted for. In this case, the greater the $\nu$, the lower the propensity to invest. Moreover, as shown in (5), the ratio $C / \bar{\Pi}_{n}^{D}$ is unaffected by the number of competing firms.

Let us next focus on the preemption hazard rate, that is, the likelihood of an event occurring in the next instant, given that the event has not occurred up to that time. In (4) the hazard rate measures the likelihood of the firm $n$ investing at $\bar{\Pi}_{n}^{D}$. Notice that $h\left(\bar{\Pi}_{n}^{D}, N\right)$ is nil when there is no probability of one firm going first, i.e., under monopoly $(N=0)$. On the other hand,

\footnotetext{
${ }^{14}$ As shown in (3) and (4), the firm's option to invest depends on $\Pi_{t}$. On the contrary, the threshold point $\bar{\Pi}_{n}^{D}$ is unaffected by current profitability, because the hazard-rate $h\left(\bar{\Pi}_{n}^{D} ; N\right)$ is independent of both $\Pi_{t}$ and $U_{t}$ (see Appendix A).

${ }^{15}$ Remember that $\varepsilon$ usually embodies tax benefits. For further details on this point see e.g., Panteghini (2007a, 2007b).
} 
it goes to infinity under perfect competition (i.e., when $N \rightarrow \infty$ ). ${ }^{16}$ In the former case, the monopolistic trigger point is equal to $\bar{\Pi}^{D}=\frac{\beta_{1}}{\beta_{1}-1} \frac{1}{1+m} \delta I_{n}$. In the latter case, the competitive (zero-NPV) trigger point is equal to $\bar{\Pi}_{N P V}^{D}=$ $\frac{1}{1+m} \delta I_{n}$. Given Proposition 1 we can thus write the following:

Corollary 1 If $h\left(\bar{\Pi}_{n}^{D} ; N\right) \in[0, \infty)$, we have $\bar{\Pi}_{N P V}^{D} \leq \bar{\Pi}_{n}^{D} \leq \bar{\Pi}^{D} \forall n$, where upper bound is reached as $h\left(\bar{\Pi}_{n}^{D} ; N\right) \rightarrow 0$, and the lower bound is obtained as $h\left(\bar{\Pi}_{n}^{D} ; N\right) \rightarrow \infty$.

Corollary 1 shows that the oligopolistic trigger point lies between the competitive (zero-NPV) and the monopolistic one. This Corollary will be necessary to compare the trade-off case, depicted in Proposition 1, with the all-equity financing one, that we are going to study. Setting $C=0$ into (4), we obtain the trigger point of a fully equity-financed firm, i.e.,

$$
\bar{\Pi}_{n}^{E}=G\left(\bar{\Pi}_{n}^{E} ; N\right) \delta I_{n}
$$

By Corollary 1, it is clear that if $h\left(\bar{\Pi}_{n}^{E} ; N\right) \in[0, \infty)$, the optimal investment strategy under all-equity lies between the competitive trigger point and the monopolistic one, i.e. $\bar{\Pi}_{N P V}^{E} \leq \bar{\Pi}_{n}^{E} \leq \bar{\Pi}^{E} \quad \forall n$, where $\bar{\Pi}_{N P V}^{E}=\delta I_{n}$ and $\bar{\Pi}^{E}=\frac{\beta_{1}}{\beta_{1}-1} \delta I_{n}$. Therefore, we can prove that:

Proposition 2 For $0<N<\infty$, we have:

$$
1>\frac{\bar{\Pi}_{n}^{D}}{\bar{\Pi}_{n}^{E}}>\frac{\bar{\Pi}^{D}}{\bar{\Pi}^{E}} .
$$

Proof. See Appendix B.

The inequality $1>\frac{\bar{\Pi}_{n}^{D}}{\bar{\Pi}_{n}^{E}}$ is the result of two offsetting effects. On the one hand, optimal leverage makes the project more profitable, thereby encouraging investment. On the other hand, financial flexibility raises the opportunity cost of investment, thus causing a delay. Proposition 2 shows that the former effect always dominates the latter: this means that financial flexibility stimulates investment, i.e., $\bar{\Pi}_{n}^{D}<\bar{\Pi}_{n}^{E}$.

The second inequality $\frac{\bar{\Pi}_{n}^{D}}{\bar{\Pi}_{n}^{E}}>\frac{\bar{\Pi}^{D}}{\bar{\Pi}^{E}}$ states that the ratio between the tradeoff and the equity-financed trigger point under preemption is greater than

\footnotetext{
${ }^{16}$ Notice that the hazard rate goes to infinity also when $U_{t}$ goes to $\bar{\Pi}^{u}$. As $U_{t} \rightarrow \bar{\Pi}^{u}$ and no firms have invested yet, each firm knows that at least one of its rivals will almost certainly act in the near future. This causes the hazard rate to explode (see LP).
} 
that obtained under monopoly $(N=0)$. This results in preemption reducing the difference between the investment strategies in the two cases under study. The reasoning behind this result is as follows. As shown by (5) the ratio $C / \bar{\Pi}_{n}^{D}$ is unaffected by strategic interactions. This implies that, under preemption, a decrease in the trigger value causes a proportional decrease in the optimal coupon $C$ and, consequently, the firms borrow a lower amount of debt. Since the incentive to borrow is reduced by preemption, the difference between the all-equity-financed and the trade-off case is smaller. In short, Proposition 2 shows that the discouraging effect of credit rationing on investment decisions is reduced by preemption.

\section{Concluding remarks}

In this article, we have provided an natural extension of existing studies, by analysing the interactions between financial and start-up decisions, in an oligopolistic market. Moreover, by comparing an all-equity-financing setup with a benchmark optimal leverage one, under incomplete information, we have analysed the effects of credit constraints on a start-up's investment strategy. As we have shown, an all-equity-financed new venture is postponed with respect to an optimally leveraged one (Proposition 1). However, we have also proven that preemption reduces the incentive to borrow. This means that, in a realistic setting where start-up firms compete to enter a new market, the discouraging effect of credit rationing on investment decision is reduced (Proposition 2). These results allow us to state that, as long as preemption exists, a limited access to credit may have negligible effects on a start-up's propensity to invest. 


\section{A Proof of Proposition 1}

To prove this Proposition, let us first calculate the firm's value function. For simplicity we set $t=0$ and, hereafter, omit the time variable. Let us define $E_{n}(\Pi ; C)$ as the market value of the equity. Following Leland (1994), its contingent claim valuation can be derived as the solution of the following equation:

$$
r E_{n}(\Pi ; C)=\Pi-(1-\varepsilon) C+(r-\delta) \Pi E_{n_{\Pi}}(\Pi ; C)+\frac{\sigma^{2}}{2} \Pi^{2} E_{n_{\Pi \Pi}}(\Pi ; C),
$$

subject to a terminal condition and to a lower boundary condition. Solving (8) we obtain:

$$
E_{n}(\Pi ; C)= \begin{cases}0 & \text { if } \quad \Pi \in[0, C), \\ \frac{\Pi}{\delta}-\frac{(1-\varepsilon) C}{r}+A_{1} \Pi^{\beta_{1}}+A_{2} \Pi^{\beta_{2}} & \text { if } \quad \Pi \in(C, \infty),\end{cases}
$$

where $\beta_{1}>0$ and $\beta_{2}<0$ are the roots of the characteristic equation $\Psi(\beta) \equiv$ $\frac{1}{2} \sigma^{2} \beta(\beta-1)+(r-\delta) \beta-r=0$.

Let us next calculate the constants $A_{1}$ and $A_{2}$. In the absence of any financial bubbles, $A_{1}$ is nil. The term $A_{2} \Pi^{\beta_{2}}$ measures the expected present value of the payoff lost after default. To calculate $A_{2}$, we apply the boundary condition $E_{n}(C ; C)=0$, which states that the value of equity is nil when default takes place. We thus obtain:

$$
E_{n}(\Pi ; C)= \begin{cases}0 & \text { if } \quad \Pi \in[0, C), \\ \frac{\Pi}{\delta}+\left\{\frac{1}{\delta}-\frac{(1-\varepsilon)}{r}\left[1-\left(\frac{\Pi}{C}\right)^{\beta_{2}}\right]\right\} C & \text { if } \quad \Pi \in(C, \infty) .\end{cases}
$$

Let us next calculate the value of debt. Similarly to equity, debt must satisfy the following differential equation:

$$
r D_{n}(\Pi ; C)=G+(r-\delta) \Pi D_{n_{\Pi}}(\Pi ; C)+\frac{\sigma^{2}}{2} \Pi^{2} D_{n_{\Pi \Pi}}(\Pi ; C),
$$

where $G=\Pi, C$. The closed-form solution of function (11) is:

$$
D_{n}(\Pi ; C)=\left\{\begin{array}{lll}
\frac{\Pi}{\delta}+B_{1} \Pi^{\beta_{1}}+B_{2} \Pi^{\beta_{2}} & \text { if } \quad \Pi \in[0, C), \\
\frac{C}{r}+D_{1} \Pi^{\beta_{1}}+D_{2} \Pi^{\beta_{2}} & \text { if } \quad \Pi \in(C, \infty) .
\end{array}\right.
$$


If the operation can be suspended when $\Pi$ falls to zero, we have $D_{n}(0 ; C)=$ 0 and the boundary condition $B_{2}=0$ is obtained. In the absence of any financial bubble, moreover, we must also have $B_{1}=D_{1}=0$. Finally, $D_{2}$ can be calculated by setting the two branches of (12), net of the default cost $v C$, meeting tangentially at point $\Pi=C .{ }^{17}$ The value of debt is therefore:

$$
D_{n}(\Pi ; C)= \begin{cases}\frac{\Pi}{\delta} & \text { if } \quad \Pi \in[0, C), \\ {\left[\frac{1}{r}+\left(\frac{1}{\delta}-\frac{1}{r}-v\right)\left(\frac{\Pi}{C}\right)^{\beta_{2}}\right] C} & \text { if } \quad \Pi \in(C, \infty) .\end{cases}
$$

Recalling (3) we must evaluate:

$$
O_{n}\left(\Pi_{t}, U_{t} ; \bar{\Pi}_{n}, C\right)=\max _{\bar{\Pi}_{n}>0, C>0}\left\{0, V_{n}\left(\Pi_{t} ; \bar{\Pi}_{n}, C\right)\left(\frac{1-F\left(\bar{\Pi}_{n} ; \Pi_{t}\right)}{1-F\left(U_{t} ; \Pi_{t}\right)}\right)^{N}\right\}
$$

with

$$
\begin{aligned}
V_{n}\left(\Pi_{t} ; \bar{\Pi}_{n}, C\right) & =E_{t}\left\{e^{-r\left(T_{n}-t\right)}\left(\int_{T_{n}}^{\infty}\left[\Pi_{s}-(1-\varepsilon) C\right] e^{-r s} d s-I_{n}\right)\right\}(15 \\
& =\left[W_{n}\left(\bar{\Pi}_{n} ; C\right)-I_{n}\right] E_{t}\left[e^{-r\left(T_{n}-t\right)}\right]
\end{aligned}
$$

where $W_{n}\left(\bar{\Pi}_{n} ; C\right)$ is firm $n$ 's static project value for $\Pi=\bar{\Pi}_{n}$. Adding (10) to (13) gives the present value of future cash flows, net of the default cost,

$$
W_{n}(\Pi ; C)=\frac{\Pi}{\delta}+\frac{C}{r}\left[\varepsilon-(\varepsilon+r \nu)\left(\frac{\Pi}{C}\right)^{\beta_{2}}\right] .
$$

Following Dixit and Pindyck (1994, pp. 315-316), it is easy to show that $E_{t}\left[e^{-r\left(T_{n}-t\right)}\right]=\left(\frac{\Pi_{t}}{\bar{\Pi}_{n}}\right)^{\beta_{1}}$. Substituting (15) and (16) into (3) we obtain

$$
\begin{aligned}
& O_{n}\left(\Pi_{t}, U_{t} ; \bar{\Pi}_{n}, C\right)= \\
& \max _{\bar{\Pi}_{n}>0, C>0}\left\{0,\left(\frac{\Pi_{t}}{\bar{\Pi}_{n}}\right)^{\beta_{1}}\left[\frac{\Pi}{\delta}+\frac{C}{r}\left(\varepsilon-(\varepsilon+r \nu)\left(\frac{\Pi}{C}\right)^{\beta_{2}}\right)-I_{n}\right]\left(\frac{1-F\left(\bar{\Pi}_{n} ; \Pi_{t}\right)}{1-F\left(U_{t} ; \Pi_{t}\right)}\right)^{N}\right\} .
\end{aligned}
$$

\footnotetext{
${ }^{17}$ For further details on these boundary conditions see e.g. Panteghini (2007a).
} 
Maximizing (17) gives the following first order conditions: ${ }^{18}$

$$
\begin{aligned}
& 0=\frac{\partial O_{n}\left(\Pi, U ; \bar{\Pi}_{n}, C\right)}{\underline{\partial}_{n}}=\frac{\partial V_{n}\left(\Pi ; \bar{\Pi}_{i}, C\right)}{\partial \bar{\Pi}_{n}} \\
& \cdot\left(1-F\left(\bar{\Pi}_{n} ; \Pi, U\right)\right)^{N}-N\left(1-F\left(\bar{\Pi}_{n} ; \Pi, U\right)\right)^{N-1} V_{n}\left(\Pi ; \bar{\Pi}_{i}, C\right) f\left(\bar{\Pi}_{n} ; \Pi, U\right),
\end{aligned}
$$

and

$$
\begin{aligned}
0 & =\frac{\partial O_{n}\left(\Pi, U ; \bar{\Pi}_{n}, C\right)}{\partial C} \\
& =\frac{\partial V_{n}\left(\Pi ; \bar{\Pi}_{i}, C\right)}{\partial C}\left(1-F\left(\bar{\Pi}_{n} ; \Pi, U\right)\right)^{N}
\end{aligned}
$$

Rearranging (18) and (19) gives the investment trigger point:

$$
\bar{\Pi}_{n}^{D}=\frac{\beta_{1}+h\left(\bar{\Pi}_{n}^{D} ; N\right)}{\beta_{1}-1+h\left(\bar{\Pi}_{n}^{D} ; N\right)} \delta I_{n}-\frac{\beta_{2}}{\beta_{2}-1} \frac{\varepsilon}{r} C \quad \forall n,
$$

with $h\left(\bar{\Pi}_{n} ; N\right) \equiv \frac{N \bar{\Pi}_{n} f\left(\bar{\Pi}_{n} ; \Pi\right)}{1-F\left(\bar{\Pi}_{n} ; \Pi\right)}$, and the ratio (5).

Substituting (5) into (20) we obtain the investment trigger point (4):

$$
\begin{aligned}
\bar{\Pi}_{n}^{D} & =\bar{\Pi}^{D}-\frac{1}{1+m} \frac{h\left(\bar{\Pi}_{n}^{D} ; N\right)}{\left(\beta_{1}-1\right)\left(\beta_{1}-1+h\left(\bar{\Pi}_{n}^{D} ; N\right)\right)} \delta I_{n} \forall n \\
& =\frac{G\left(\bar{\Pi}_{n}^{D} ; N\right)}{1+m} \delta I_{n},
\end{aligned}
$$

where $G\left(\bar{\Pi}_{n}^{D} ; N\right) \equiv \frac{\beta_{1}+h\left(\bar{\Pi}_{n}^{D} ; N\right)}{\beta_{1}-1+h\left(\bar{\Pi}_{n}^{D} ; N\right)}$. Note that, given $(2)$, the hazard rate is unaffected by downward truncations of the distribution $F\left(\bar{\Pi}_{n} ; \Pi\right)$, i.e.,

$$
h\left(\bar{\Pi}_{n} ; N\right)=\frac{N \bar{\Pi}_{n} f_{t}\left(\bar{\Pi}_{n} ; U_{t}\right)}{1-F_{t}\left(\bar{\Pi}_{n} ; U_{t}\right)}=\frac{N \bar{\Pi}_{n} f\left(\bar{\Pi}_{n} ; \Pi\right)}{1-F\left(\bar{\Pi}_{n} ; \Pi\right)},
$$

This makes the optimal operating rule (4) stationary. Proposition 1 is thus proven.

\footnotetext{
${ }^{18}$ It is easy to show that a sufficient, although not necessary, condition for the second order condition $\frac{\partial^{2} O_{n}\left(\Pi, U ; \bar{\Pi}_{n}, C\right)}{\partial\left(\bar{\Pi}_{n}\right)^{2}}<0$ to hold is that $h\left(\bar{\Pi}_{n}^{D} ; N\right)$ is increasing in $\bar{\Pi}_{n}^{D}$. The positive derivative is a common feature of standard distributions.
} 


\section{B Proof of Proposition 2}

Let us start with the oligopolistic case. Using (4) and (6) we obtain:

$$
\frac{\bar{\Pi}_{n}^{D}}{\bar{\Pi}_{n}^{E}}=\frac{1}{1+m} \frac{G\left(\bar{\Pi}_{n}^{D} ; N\right)}{G\left(\bar{\Pi}_{n}^{E} ; N\right)} .
$$

We can prove that

$$
\frac{\bar{\Pi}_{n}^{D}}{\bar{\Pi}_{n}^{E}}<1
$$

by contradiction. Let us assume ab absurdo that $\frac{\bar{\Pi}_{n}^{D}}{\bar{\Pi}_{n}^{E}}>1$. Since $\frac{\partial G(\bar{\Pi} ; N)}{\partial \bar{\Pi}} \propto$ $-\frac{\partial h(\bar{\Pi} ; N)}{\partial \bar{\Pi}}$, we have $G\left(\bar{\Pi}_{n}^{D} ; N\right)<G\left(\bar{\Pi}_{n}^{E} ; N\right)$ : this contradicts $(24)$.

Let us next turn to the monopolistic case. Setting the hazard rate equal to zero, i.e., $h(\bar{\Pi}, 0)=0 \forall \bar{\Pi}$, gives the trigger points under optimal leverage, i.e., $\bar{\Pi}^{D}=\frac{\beta_{1}}{\beta_{1}-1} \frac{\delta I_{n}}{1+m}$, and under all-equity financing, i.e., $\bar{\Pi}^{E}=$ $\frac{\beta_{1}}{\beta_{1}-1} \delta I_{n}$, respectively. Next, calculate the ratio:

$$
\frac{\bar{\Pi}^{D}}{\bar{\Pi}^{E}}=\frac{1}{1+m}<1 .
$$

Compare now (23) with (25). Since $G\left(\bar{\Pi}_{n}^{D} ; N\right)>G\left(\bar{\Pi}_{n}^{E} ; N\right)$, we have

$$
\frac{\bar{\Pi}_{n}^{D}}{\bar{\Pi}_{n}^{E}}>\frac{\bar{\Pi}^{D}}{\bar{\Pi}^{E}} .
$$

Using (24) and (26) we thus obtain (7). This proves Proposition 2.

\section{References}

[1] Coy P. (1999), Exploiting Uncertainty: The Real Option Revolution in Decision-Making, Business Week, June 7.

[2] Dixit A. and R.S. Pindyck (1994), Investment under Uncertainty, Princeton University Press, Princeton.

[3] Dosi C. and M. Moretto (2007), Environmental Innovation, War of Attrition and Investment Grants, International Game Theory Review, (forthcoming). 
[4] Gamba A., G. Sick. and M. Aranda (2005), Investment under Uncertainty, Capital Structure and Taxes, Available at SSRN: http://ssrn.com/abstract $=676572$.

[5] Graham J.R. and C.R. Harvey (2001), The Theory and Practice of Corporate Finance: Evidence from the Field, Journal of Financial Economics, 60, pp. 187-243.

[6] Grenadier S.R. (2002), Option Exercise Games: An Application to the Equilibrium Investment Strategies of Firms, The Review of Financial Studies, 15, pp. 691-721.

[7] Huisman K.J.M. (2001), Technology Investment: A Game Theoretical Real Options Approach, Kluwer Academic Publisher, Boston.

[8] Jagannathan R. and I. Meter, (2002), Do We Need CAPM for Capital Budgeting?, NBER Working Paper n. 8719.

[9] Jensen M. (1986), Agency Costs of Free Cash Flow, Corporate Finance, and Takeovers, American Economic Review, 76, pp. 323-329.

[10] Lambrecht B.M. (2001), The Impact of Debt Financing on Entry and Exit in a Duopoly, The Review of Financial Studies, 14, pp. 765-804.

[11] Lambrecht B. and W. Perraudin, (2003), Real Options and Preemption under Incomplete Information, Journal of Economic Dynamics and Control, 27, pp.619-643.

[12] Leland H.E. (1994), Corporate Debt Value, Bond Covenants, and Optimal Capital Structure, Journal of Finance, 49, pp. 1213-1252.

[13] Mauer D.C. and S.H. Ott (2000), Agency Costs, Underinvestment, and Optimal Capital Structure, in M.J. Brennan and L. Trigeorgis (eds.), Perfect Flexibility, Agency, and Competition, New Developments in the Theory and Application of Real Options, Oxford University Press, New York.

[14] McDonald R.L. (2000), Real Options and Rules of Thumb in Capital Budgeting, in M.J. Brennan and L. Trigeorgis (editors), Project Flexibility, Agency, and Competition, New Developments in the Theory and Application of Real Options, Oxford University Press, New York. 
[15] McDonald R. and D. Siegel (1985), Investment and the Valuation of Firms When There is an Option to Shut Down, International Economic Review, 26, pp. 331-349.

[16] Mello A.S. and J.E. Parsons (1992), Measuring the Agency Cost of Debt, Journal of Finance, 47, pp. 1887-1903.

[17] Miao J. (2005), Optimal Capital Structure and Industry Dynamics, Journal of Finance, 60, pp. 2621-2659.

[18] Moretto M. (2000), Irreversible Investment with Uncertainty and Strategic Behavior, Economic Modelling, 17, pp. 589-617.

[19] Ottoo R.E. (2001), Valuation of Corporate Growth Opportunities: A Real Option Approach, Garland Publishing, Inc., New York.

[20] Panteghini P.M. (2007a), Corporate Taxation in a Dynamic World, Springer.

[21] Panteghini P.M. (2007b), Interest Deductibility under Default Risk and the Unfavorable Tax Treatment of Investment Costs: A Simple Explanation, Economics Letters, forthcoming.

[22] Smit H.T.J. and L. Trigeorgis (2004), Strategic Investment Real Option and Games, Princeton University Press, Princeton. 